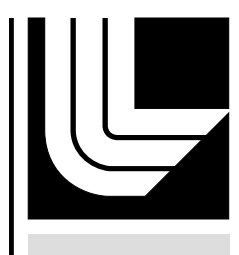

LAW RENCE LIVERMORE N A TION A L LABORATORY

Low Frequency Electromagnetic Pulse and Explosions

J. J. Sweeney

March 3, 2011 
This document was prepared as an account of work sponsored by an agency of the United States government. Neither the United States government nor Lawrence Livermore National Security, LLC, nor any of their employees makes any warranty, expressed or implied, or assumes any legal liability or responsibility for the accuracy, completeness, or usefulness of any information, apparatus, product, or process disclosed, or represents that its use would not infringe privately owned rights. Reference herein to any specific commercial product, process, or service by trade name, trademark, manufacturer, or otherwise does not necessarily constitute or imply its endorsement, recommendation, or favoring by the United States government or Lawrence Livermore National Security, LLC. The views and opinions of authors expressed herein do not necessarily state or reflect those of the United States government or Lawrence Livermore National Security, LLC, and shall not be used for advertising or product endorsement purposes.

This work performed under the auspices of the U.S. Department of Energy by Lawrence Livermore National Laboratory under Contract DE-AC52-07NA27344. 


\section{Low Frequency Electromagnetic Pulse and Explosions}

Jerry Sweeney, Lawrence Livermore National Laboratory

LLNL-TR-??????

January 5, 2011

\section{Summary}

This paper reviews and summarizes prior work related to low frequency $(<100 \mathrm{~Hz})$ EMP (ElectroMagnetic Pulse) observed from explosions. It focuses on how EMP signals might, or might not, be useful in monitoring underground nuclear tests, based on the limits of detection, and physical understanding of these signals. In summary:

- Both chemical and nuclear explosions produce an EMP.

- The amplitude of the EMP from underground explosions is at least two orders of magnitude lower than from above ground explosions and higher frequency components of the signal are rapidly attenuated due to ground conductivity.

- In general, in the near field, that is distances (r) of less than 10 s of kilometers from the source, the amplitude of the EMP decays approximately as $1 / \mathrm{r}^{3}$, which practically limits EMP applications to very close $(<\sim 1 \mathrm{~km})$ distances.

- One computational model suggests that the EMP from a decoupled nuclear explosion may be enhanced over the fully coupled case. This has not been validated with laboratory or field data.

- The magnitude of the EMP from an underground nuclear explosion is about two orders of magnitude larger than that from a chemical explosion, and has a larger component of higher frequencies. In principle these differences might be used to discriminate a nuclear from a chemical explosion using sensors at very close $(<\sim 1 \mathrm{~km})$ distances.

- Arming and firing systems (e.g. detonators, exploding bridge wires) can also produce an EMP from any type of explosion.

- To develop the understanding needed to apply low frequency EMP to nuclear explosion monitoring, it is recommended to carry out a series of controlled underground chemical explosions with a variety of sizes, emplacements (e.g. fully coupled and decoupled), and arming and firing systems. 


\section{Background}

The first published accounts of measurements of electromagnetic pulse (EMP) signals from chemical explosions appeared in the 1950s. Since then, numerous studies have been carried out confirming the initial results and better characterizing the phenomenon. General opinion is that the EMP from chemical explosions is caused by charge separation of explosive products after detonation. Movement of charges above the relatively conductive ground surface results in a changing electric charge dipole. Relative rotational movements of charges in the debris cloud are thought to be the source of the magnetic field. Other EMP effects are caused by the reflection of the shock wave from the ground surface and interaction with the debris cloud. It is also well known that electronic detonators create an EMP. The amplitude of the different types of EMP signals that are observed is directly proportional to the mass of explosive, the character of the explosive (e.g., presence of conductors in the explosive or from metallic casing), height of burst above ground, and distance and azimuth where the measurement is carried out. Differences in the observed dependence of field strength that depend on range depend on frequency and may be due to whether the range is in the static $\left(1 / \mathrm{r}^{3}\right.$ dependence), induction $\left(1 / \mathrm{r}^{2}\right.$ dependence), or far field ( $1 / \mathrm{r}$ dependence) zone. At least one study has suggested a quadrupole source for explosions above the ground surface $\left(1 / \mathrm{r}^{4}\right.$ dependence). Also to be noted is that a delay has been observed, on the order of $10-20 \mathrm{~ms}$, in the development of the EMP after initiation of the detonation. This is likely related to the explosion initiation time.

EMP from nuclear explosions was noted and studied starting from the initial development of nuclear weapons. The phenomenon of EMP from above ground and atmospheric nuclear explosions has been extensively studied, including the weapons effects of the EMP. There have been only a few studies in the open literature of EMP from both chemical and nuclear explosions detonated underground. In the late 1980s, LLNL carried out a campaign of field measurements (Sweeney, 1989) to observe low frequency (less than $100 \mathrm{~Hz}$ ) EMP at ranges of several kilometers from surface ground zero (SGZ) of underground nuclear explosions (UNEs). In this study, measurements were made at up to three different locations up to $15 \mathrm{~km}$ from SGZ from 17 different UNEs. A strong low frequency EMP was observed for many of these explosions, but not enough data were available to systematically estimate dependence of the EMP on yield, depth of burial, distance, azimuth, or other factors. The result of this study was the observation that the EMP could not be observed at distances more than $10 \mathrm{~km}$ from ground zero for UNEs with yields up to $150 \mathrm{kt}$. An additional observation was that the EMP from a UNE does not couple into the earth-ionosphere electrical cavity as well as impulses from worldwide lightning activity.

LLNL also carried out measurements of low frequency EMP during the Nonproliferation Experiment (NPE), in which approximately $1 \mathrm{kt}$ of chemical explosive was detonated underground at Rainier Mesa at the Nevada Test Site (Sweeney, 
1995). Low frequency EMP was detected at two locations with slant range of 500 and $1000 \mathrm{~m}$ from this chemical explosion. A comparison of the EMP from the NPE with the EMP measured at a similar distance from a nuclear explosion carried out in the same area (refer to figure below) reveals important differences in the character of the low frequency EMP. The EMP from the UNE has a very steep rise time, a short pulse duration, and there is no delay from the detonation time. The EMP from the chemical explosion has a much more gradual rise time, longer pulse duration, and a delay of $19 \mathrm{~ms}$ between detonation time and initiation of the EMP. The rise in the measured cavity pressure during the NPE is similar to the rise in the EMP as shown in the figure; this suggests that the chemical EMP is driven by development of the explosion products, as is seen in the case of above ground chemical explosions. The sharp rise time of the EMP in the UNE case is caused by the well-understood production of Compton electrons resulting from the nuclear fission. These observed differences suggest that low frequency EMP could be used to distinguish between chemical and nuclear explosions.

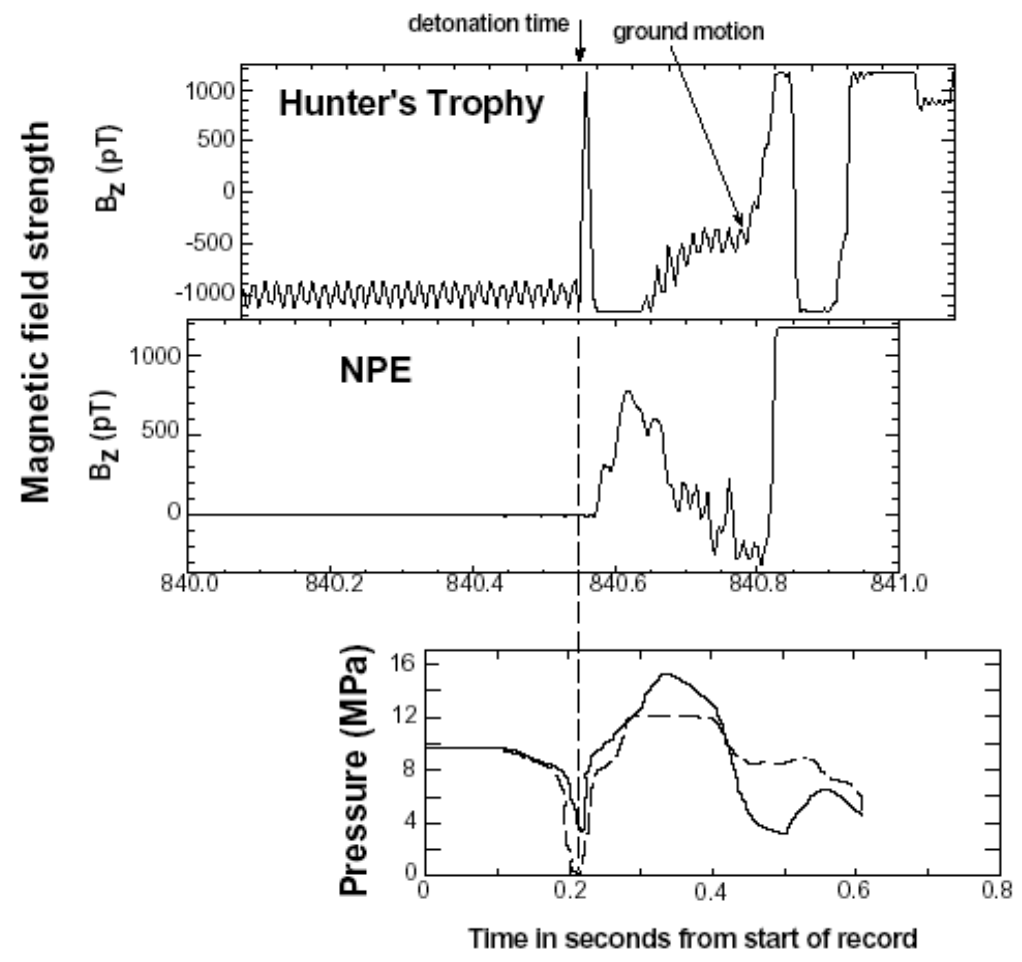

Vertical magnetic signals recorded during the Hunter's Trophy underground nuclear explosion (top) and the nonproliferation experiment (NPE) chemical explosion (middle). The bottom figure is a plot of cavity pressure recorded during the NPE versus time. The horizontal scale in all three plots is time in seconds. The similar arrival of the ground motion signals of the top two plots results from the similar range (about $500 \mathrm{~m}$ ) to the sensors.

In the late 1990s, LLNL personnel collaborated with Russian Federation scientists from the All-Russian Institute for Nuclear Physics (VNIITF) in Snezhinsk on an International Science and Technology Center (ISTC) project to systematically study 
EMP from chemical explosions. In this study, a variety of measurement systems, covering frequency ranges from $10 \mathrm{~s}$ of $\mathrm{Hz}$ to $\mathrm{MHz}$ were used to measure EMP at distances of up to tens of meters from explosions in the air, at the ground surface, underground, and in an underground cavity at yields of up to $500 \mathrm{Kg}$. Two papers from this work (Soloviev, et al., 2002 and Soloviev and Sweeney, 2005) showed that both magnetic and electrical EMP are generated from explosions underground and in boreholes and that in the near field (much less than one wavelength measurement range) the EMP can be modeled as a changing charge quadrupole, with amplitude showing a $1 / \mathrm{r}^{4}$ decay with distance.

Another result from the VNIITF collaboration and ISTC funding was publication in the west of two papers by scientists from the VNIITF (Kovalenko et al., 2001 and Karlykhanov, 2004) resulting from efforts to model the EMP from UNEs. In the first case, the EMP was modeled for a fully contained and coupled $1 \mathrm{kt}$ chemical and nuclear explosions underground. The second paper dealt with the interesting case of a $1 \mathrm{kt}$ decoupled nuclear explosion detonated underground in a cavity. In both cases, the explosions are modeled as an expanding conducting explosion products subject to the different rise time and temperature conditions for the differing chemical and nuclear cases. Conductivities are smaller for the chemical explosion case, giving rise to magnetic fields that are about two orders of magnitude smaller than the nuclear case. As the conductive shell expands within the ambient earth magnetic field the field lines are distorted (also called a magnetic "bubble"). The EMP results from the disturbance to the ambient field as the field is perturbed and then relaxes back into the steady state once the source of charge (due to cooling or end of intense fission) disappears. In the first case, the calculations suggest that the observed differences in EMP (the papers cite the NPE example rather than any Russian measurements) between underground chemical and nuclear explosions are caused by the fundamental differences in the nature of the nuclear and chemical reactions that cause the explosions. In the decoupled case, the modeling makes the intriguing suggestion that the EMP for a decoupled UNE could be enhanced over the fully coupled case. The difference comes from the larger radius of the sphere of charge in a cavity and by inference, would hold for a chemical explosion as well. These are significant results because, with the exception of direct measurement of neutron flux or later radiochemistry measurements, there is at present no other way to distinguish between chemical and nuclear explosions and EMP measurements could be a means to determine whether seismic energy from the explosion has been damped by decoupling.

To summarize:

- Both chemical and nuclear explosions produce an EMP.

- The amplitude of the EMP from underground explosions is at least two orders of magnitude lower than from above ground explosions. Because of ground conductivity, higher frequency components of the signal are rapidly attenuated (the skin effect).

- One computational model suggests that the EMP from a decoupled nuclear explosion may be enhanced over the fully coupled case. 
- The magnitude of the EMP from a UNE is about two orders of magnitude larger than that from a chemical explosion, and EMP from a UNE has a larger component of higher frequencies.

- In general, in the near field (ranges of less than 10s of kilometers) the amplitude of the EMP decays approximately as $1 / \mathrm{r}^{3}$.

- Arming and firing systems (e.g. detonators, exploding bridge wires) can also produce an EMP.

The critical factor determining usefulness of EMP measurements for explosion monitoring is the distance dependence of the phenomena. The initial studies of UNEs suggested a $1 / \mathrm{r}^{2}$ to $1 / \mathrm{r}^{3}$ signal fall-off with distance. The table below provides conservative estimates of the minimum measurement range from the explosion needed to detect the EMP for underground chemical and nuclear explosions of various yields. It should be noted that these estimates are based on ideal signal-tonoise conditions. In the context of explosion monitoring for the Comprehensive Nuclear Test Ban Treaty (CTBT), the close monitoring ranges of the table mean that use of EMP is confined to use as a transparency method. The potential ability to determine whether the explosion is nuclear or chemical and estimate the level of decoupling is very attractive in this context because EMP methods may be considered to be less intrusive than direct nuclear measurements (which would have to be done even closer to the source). An added benefit of an EMP measurement is that it provides an explosion origin time (within ms) that is useful for seismic location determinations.

\begin{tabular}{|l|l|l|}
\hline Yield & Max. range, meters & Max. range, meters \\
\hline & Chemical explosion & Nuclear explosion \\
\hline & & \\
\hline $10 \mathrm{~kg}$ & $9-12$ & $41-54$ \\
\hline $100 \mathrm{~kg}$ & $19-25$ & $87-116$ \\
\hline 1 ton & $41-54$ & $188-250$ \\
\hline 10 tons & $87-116$ & $405-539$ \\
\hline 100 tons & $188-250$ & $874-1160$ \\
\hline
\end{tabular}

\section{Practical issues}

It is important to note that the comparison table above is empirical and based on a very small amount of data, with only one direct comparison between a UNE and an underground chemical explosion. There are no low frequency EMP data from large decoupled chemical explosions, so at this point it is not possible to make any quantitative evaluation of decoupling using EMP. Practical application of low frequency EMP at this time can only be used for discrimination (by using the rise time of the prompt low frequency EM signal). Because there are no validated models of the underground EMP from chemical or nuclear explosions, it is not possible at this time to use signal magnitude (which depends on range, azimuth, explosion 
characteristics, yield, and geologic factors) as a discriminant nor as a way of estimating yield. It should also be noted that much more study is needed of the interfering signals related to arming and firing systems and at what range these become a problem. Typical shock wave arrival propagation velocities are about $2000 \mathrm{~m} / \mathrm{s}$. At a range of $100 \mathrm{~m}$, this allows only $5 \mathrm{~ms}$ for an EMP measurement before the ground shock arrives at the sensor. This could be a possible problem for monitoring at the lowest yields at close range. Finally, the environment for the type of experiments of interest to transparency will be rich in industrial power EM signals. For adequate monitoring of low frequency EMP, extensive electronic filtering will be needed (e.g. notch filters) to remove AC power line frequencies (fundamental and multiples).

\section{Recommendations and suggestions for further work}

The most effective way to enhance understanding of the application of low frequency EMP for transparency will be to carry out a series of controlled experiments with underground fully coupled and decoupled explosions with a variety of chemical yields. Smaller yields are inherently difficult because of short time arrival of the ground shock, which affects the EMP sensors, so yields of 1 ton or larger are probably desirable. If electronic ignition of the explosions is used, the EMP from these systems should be well understood, otherwise non-electronic ignition should be used. It will also be important to obtain the detonation origin time (ms accuracy), preferably by means of an optical fiber measurement. Currently, magnetometer instrumentation is available and adequate for measuring magnetic field changes at frequencies of $10 \mathrm{KHz}$ and lower, but the electric field measurements are more difficult - especially the vertical electric field - and these would need some effort in sensor development. Seismic digital recording systems for these types of measurements can be used, but they are confined to sampling rates of $500 \mathrm{~Hz}$ or less. Some effort would be needed to develop field-ready digital recorders for higher sampling rates. The SPE experiments being planned by NSTech under the NCNS program would be an excellent opportunity to begin testing EMP sensor and recording systems that will be essential for success of later dedicated experiments.

\section{References}

Sweeney, J. J., 1989, An Investigation of the Usefulness of Extremely Low-Frequency Electromagnetic Measurements for Treaty Verification, Lawrence Livermore National Laboratory report UCRL-53899, 59 pp.

Sweeney, J. J., 1995, Low-Frequency Electromagnetic Measurements at the NPE and Hunter's Trophy: A Comparison, in Proceedings of the Symposium on the NonProliferation Experiment, April 19-21, 1994, Rockville, MD, US Dept. of Energy document CONF-9404100, pp. 8-21 to 8-33. 
Soloviev, S. P., V. V. Surkov, and J. J. Sweeney, 2002, Quadrupolar electromagnetic field from detonation of high explosive charges on the ground surface, J. Geophys. Res., V. 107, no. B6, pp. 2119-2130.

Soloviev, S. P., and J. J. Sweeney, 2005, Generation of electric and magnetic field during detonation of high explosive charges in boreholes, J. Geophys. Res., V. 110, B01312, doi: 10.1029/2004JB003223.

Kovalenko, G. V., A. A. Kondrat'ev, Yu. I. Matveenko, V. N. Nogin, and A. V. Petrovtsev, 2001, Magnetic dipole moment produced by an explosion of one kiloton of TNT, J. Appl. Mech. and Tech. Physics, V. 42, no. 3, pp. 387-391.

Karlykhanov, N. G., A. A. Kondrat'ev, Yu. I. Matveenko, and V. N. Nogin, 2004, Magnetic dipole moment from a one-kiloton underground nuclear explosion in a cavity, J. Appl. Mech. and Tech. Physics, V. 45, no. 3, pp. 311-315. 